Document downloaded from:

http://hdl.handle.net/10251/40600

This paper must be cited as:

Rivera Ortun, MJ.; Trujillo Guillen, M.; Romero García, V.; López Molina, JA.; Berjano Zanón, E. (2013). Numerical resolution of the hyperbolic heat equation using smoothed mathematical functions instead of Heaviside and Dirac delta distributions. International Communications in Heat and Mass Transfer. 46:7-12.

doi:10.1016/j.icheatmasstransfer.2013.05.017.

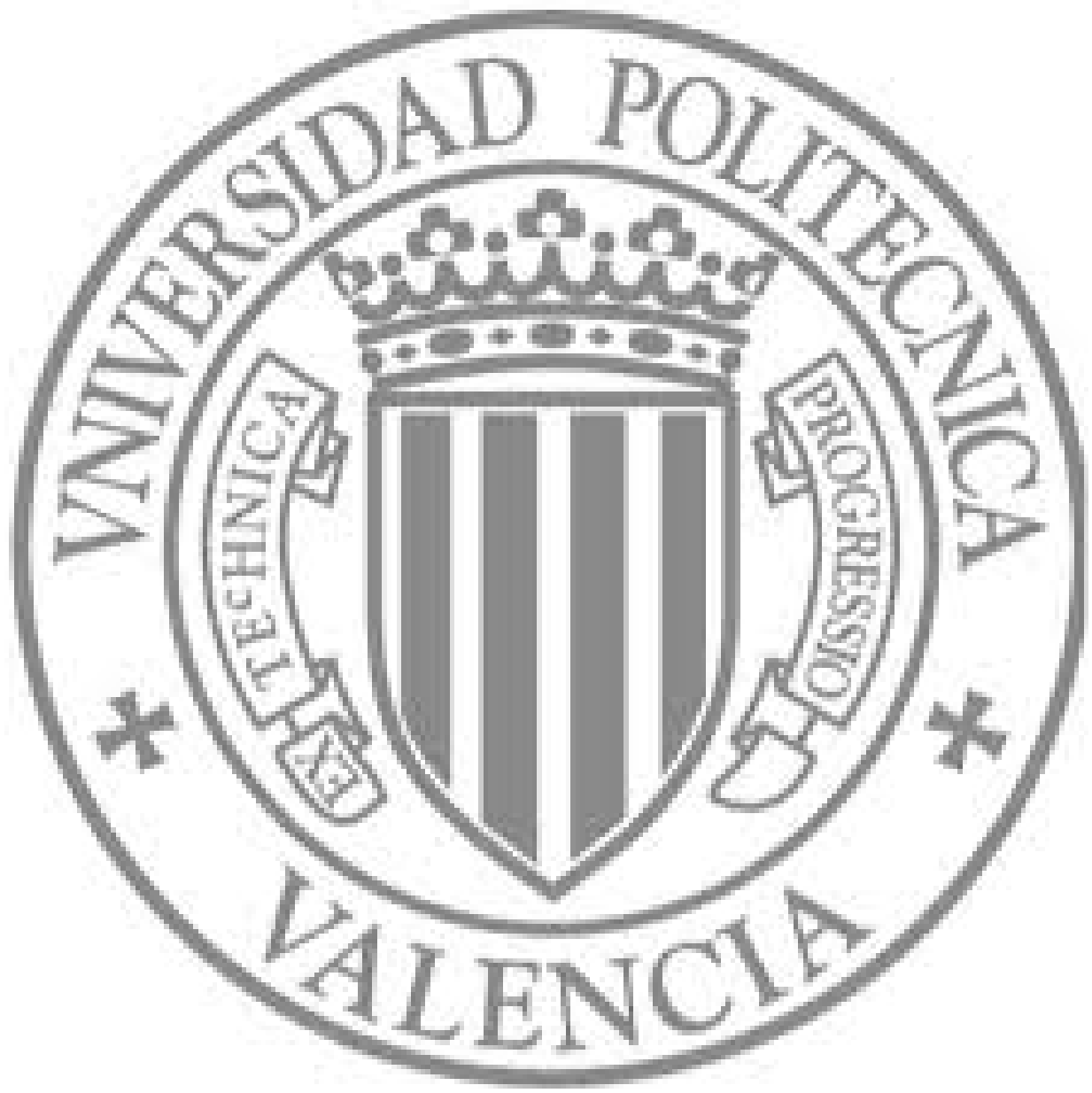

The final publication is available at

http://dx.doi.org/10.1016/j.icheatmasstransfer.2013.05.017

Copyright Elsevier 


\title{
Numerical resolution of the hyperbolic heat equation using smoothed mathematical functions instead of heaviside and Dirac delta distributions
}

\author{
M. J. Rivera ${ }^{1}$, M. Trujillo ${ }^{1}$, V. Romero-García ${ }^{2}$, J. A. López Molina ${ }^{1}$ and \\ E. Berjano ${ }^{3 \sharp}$
}

${ }^{1}$ Instituto Universitario de Matemática Pura y Aplicada, Universitat Politècnica de València, Valencia, Spain

${ }^{2}$ Instituto de Investigación para la Gestión Integrada de zonas Costeras (IGIC), Universitat Politècnica de València, Valencia, Spain

${ }^{3}$ Biomedical Synergy. Electronic Engineering Department, Universitat Politècnica de València, Valencia, Spain

$\sharp$ Address for correspondence:

Dr. Enrique Berjano

Biomedical Sinergy

Electronic Engineering Department (7F)

Universitat Politècnica de València

Camino de Vera, 46022 Valencia, Spain

E-mail: eberjano@eln.upv.es

Acknowledgements: This work received financial support from the Spanish "Plan Nacional de I+D+I del Ministerio de Ciencia e Innovación” Grant No. TEC2011-27133C02-01 and from Universitat Politènica de València (PAID-06-11 Ref. 1988). V. Romero García is grateful for the support of "Programa de Contratos Post-Doctorales con Movilidad UPV del Campus de Excelencia (CEI-01-11)" and FEDER Project MAT2009-09438. 


\begin{abstract}
The hyperbolic bioheat equation (HBE) has been used to model heating applications involving very short power pulses. This equation includes two mathematical distributions (Heaviside and Delta) which have to be necessarily substituted for smoothed mathematical functions when the HBE is solved by numerical methods. This study focuses on which type of smoothed functions would be suitable for this purpose, i.e. those which would provide provide solutions similar to those obtained analytically from the original Heaviside and Delta distributions. The logistic function was considered as a substitute for the Heaviside function, while its derivative and the probabilistic Gaussian function were considered as substitutes for the Delta distribution. We also considered polynomial interpolation functions, in particular, the families of smoothed functions with continuous second derivative without overshoot used by COMSOL Multiphysics. All the smoothed functions were used to solve the HBE by the Finite Element Method (COMSOL Multiphysics), and the solutions were compared to those obtained analytically from the original Heaviside and Delta distributions. The results showed that only the COMSOL smoothed functions provide a numerical solution almost identical to the analytical one. Finally, we demonstrated mathematically that in order to find a suitable smoothed function $(f)$ must adequately substitute any mathematical distribution $(D)$ in the HBE, the difference $D-f$ must have compact support.
\end{abstract}

Keywords: Dirac distribution, numerical method, Heaviside distribution, hyperbolic bioheat equation, non-Fourier heat, wave heat, radiofrequency ablation. 


\section{Introduction}

Mathematical modeling is widely used to study temperature distributions in different medical procedures such as radiofrequency (RF) thermal ablation [1]. Most modeling studies use the bioheat equation (BE), which is based on Fourier's Law [2]:

$$
-\Delta T(\mathbf{x}, t)+\frac{1}{\alpha} \frac{\partial T}{\partial t}(\mathbf{x}, t)=\frac{1}{k} S(\mathbf{x}, t)
$$

where $T(\mathbf{x}, t)$ and $S(\mathbf{x}, t)$ are respectively the temperature and the internal heat sources at point $\mathbf{x}$ at time $t, k$ is thermal conductivity and $\alpha$ is thermal diffusivity ( $\alpha=\frac{k}{\eta c}, \eta$ being density and $c$ specific heat). When the BE is used to study thermal therapies, the source term $S(\mathbf{x}, t)$ usually includes a heat source produced by an external energy source $S_{s}(x, t)$ (e.g. laser, microwave, ultrasound or radiofrequency) and a blood perfusion term $S_{p}(\mathbf{x}, t)$. The BE assumes an infinite thermal energy propagation speed, i.e. any local temperature disturbance causes an instantaneous perturbation in the temperature at every point in the medium [3]. Although the BE can be valid in most heat modeling, under certain circumstances, such as very fast heating with short power pulses, a model which considers a finite thermal energy propagation speed has been found to be more accurate [4]. For this reason, some modeling studies ([3]-[15]) employed the hyperbolic (non-Fourier or wave) bioheat equation (HBE) which has a double-derivative term (called the wave term) that changes BE to a hyperbolic partial differential equation [4]:

$$
-\Delta T(\mathbf{x}, t)+\frac{1}{\alpha}\left(\frac{\partial T}{\partial t}(\mathbf{x}, t)+\tau \frac{\partial^{2} T}{\partial t^{2}}(\mathbf{x}, t)\right)=\frac{1}{k}\left(S(\mathbf{x}, t)+\tau \frac{\partial S}{\partial t}(\mathbf{x}, t)\right),
$$

in which there is a new parameter $\tau$, which is the thermal relaxation time of the tissue. This equation therefore assumes a finite heat conduction speed [16], which is inversely proportional to $\tau$.

We previously developed mathematical models using HBE to study the temperature distributions in RF ablation (RFA) [17, 18]. To achieve an analytical solution, we considered the simplest geometry, consisting of a spherical active electrode with a radius of $r_{0}$ completely embedded in the biological tissue. The tissue was considered homogeneous with infinite dimension and the dispersive electrode placed at infinity. For this geometry model we used spherical coordinates, and since the model presented radial symmetry, a one-dimensional approach was possible, $r$ being the dimensional variable. Consequently, the model domain was restricted to the biological tissue, i.e. the electrode body was not included. 
From the point of view of the HBE model, Equation (2) in spherical coordinates constitutes the governing equation, where $k, \eta, c$ and $\tau$ are assumed to be constants. The source term for the RFA is the Joule heat produced per unit volume of tissue, $\left(S_{s}(r, t)\right)$, which can be expressed as [19]:

$$
S_{s}(r, t)=\frac{P r_{0}}{4 \pi r^{4}} H(t)
$$

where $P$ is the total applied power $(W)$ and $H(t)$ is the Heaviside distribution, which allows the constant power application to be modeled by means of a step at $t=0$. The blood perfusion term can be expressed as [2]:

$$
S_{p}(r, t)=-\eta_{b} c_{b} w_{b}\left(T-T_{0}\right),
$$

where $\eta_{b}$ is blood density, $c_{b}$ is the blood specific heat, $w_{b}$ is the perfusion coefficient and $T_{0}$ is the blood temperature. Then, the internal heat source term is

$$
S(r, t)=\frac{P r_{0}}{4 \pi r^{4}} H(t)-\eta_{b} c_{b} w_{b}\left(T(r, t)-T_{0}\right),
$$

and

$$
S(r, t)+\tau \frac{\partial S}{\partial t} S(r, t)=\frac{P r_{0}}{4 \pi r^{4}}(H(t)+\tau \delta(t))-\eta_{b} c_{b} w_{b} \frac{\partial T}{\partial t} T(r, t)
$$

where $\delta(t)$ is the Dirac delta distribution. Accordingly, Equation (2) in spherical coordinates is:

$$
-\alpha\left(\frac{\partial^{2} T}{\partial r^{2}}(r, t)+\frac{2}{r} \frac{\partial T}{\partial r}(r, t)\right)+\zeta \frac{\partial T}{\partial t}(r, t)+\tau \frac{\partial^{2} T}{\partial t^{2}}=\frac{P \alpha r_{0}}{4 \pi k r^{4}}(H(t)+\tau \delta(t))-B\left(T-T_{0}\right),
$$

where $B=\frac{\alpha \eta_{b} c_{b} w_{b}}{k}$ and $\zeta=1+\tau B$.

The initial conditions are

$$
T(r, 0)=T_{0}, \quad \frac{\partial T}{\partial t}(r, 0)=0 \quad \forall r>r_{0} .
$$

The boundary condition at infinity is

$$
\lim _{r \rightarrow \infty} T(r, t)=T_{0} \quad \forall t>0 .
$$

To write the boundary condition at $r=r_{0}$ (interface electrode-tissue), we adopt a simplification assuming thermal conductivity of the electrode to be greater than that 
of the tissue, which is generally true $(15 \mathrm{~W} / \mathrm{K} \cdot \mathrm{m}$ in metal vs $0.5 \mathrm{~W} / \mathrm{K} \cdot \mathrm{m}$ in tissue), i.e. considering that the boundary condition at the electrode-tissue interface is mainly governed by the thermal inertia of the electrode [19]. Thus, at each time $t$, the heat flux along the electrode surface per unit time is inverted to produce a heat increment in the mass electrode:

$$
\eta_{0} c_{0} \frac{4 \pi r_{0}^{3}}{3} \frac{\partial T}{\partial t}\left(r_{0}, t\right)
$$

where $\eta_{0}$ and $c_{0}$ are respectively the density and specific heat of the active electrode. Using the expression for the hyperbolic heat flux [4] we obtained

$$
\eta_{0} c_{0} \frac{4 \pi r_{0}^{3}}{3} \frac{\partial T}{\partial t}\left(r_{0}, t\right)=4 \pi r_{0}^{2} \frac{k}{\tau} e^{-\frac{t}{\tau}} \int_{0}^{t} e^{\frac{\psi}{\tau}} \frac{\partial T}{\partial r}\left(r_{0}, \psi\right) d \psi
$$

and by derivation with respect to $t$ we obtained the remaining boundary condition

$$
\frac{\tau \eta_{0} c_{0} r_{0}}{3 k}\left(\frac{1}{\tau} \frac{\partial T}{\partial t}\left(r_{0}, t\right)+\frac{\partial^{2} T}{\partial t^{2}}\left(r_{0}, t\right)\right)=\frac{\partial T}{\partial r}\left(r_{0}, t\right) .
$$

The majority of heat transfer problems that arise from real situations either involve complex geometries, are non-linear, or their boundary conditions lead us to use numerical instead of analytical methods to solve them. For instance, the Finite Elements Method (FEM) has been used to solve many RFA mathematical models [20]-[23]. In this respect, it is crucial to note that prior using a numerical method to solve the HBE, it is necessary to substitute the Heaviside and Dirac distributions $(H(t)$ and $\delta(t))$ for smoothed functions, which can be addressed by FEM. It is also necessary to emphasize that the Heaviside function $H(t)$ is a non continuous function and that the Dirac's Delta $\delta(t)$ is a distribution but not a function, it is in fact a measure. The annex provides information about these two distributions and the underlying theory, Schwartz's Distributions Theory.

The aim of this study was to mathematically justify the type of smoothed functions that can substitute the Heaviside and Delta distributions in the HBE and reach solutions identical to those obtained with the original distributions. We consider that this information is crucial when the HBE has to be solved by numerical methods, since substitution is mandatory. As far as we know no previous studies have addressed this issue.

\section{Materials and methods}

\section{II.1 Analytical solution of the HBE and conditions of substitution}

First we consider a general formulation of Equation (6):

$$
-\alpha\left(\frac{\partial^{2} T}{\partial r^{2}}(r, t)+\frac{2}{r} \frac{\partial T}{\partial r}(r, t)\right)+\zeta \frac{\partial T}{\partial t}(r, t)+\tau \frac{\partial^{2} T}{\partial t^{2}}=\frac{P \alpha r_{0}}{4 \pi r^{4}} D(t)-B\left(T-T_{0}\right),
$$


with conditions (7)-(11) and where $D(t)$ is any function of $t$.

The analytical solution of this problem is denoted by $T(r, t, D(t))$. Our goal is to mathematically compare the analytical solutions $T(r, t, H(t)+\tau \delta(t))$ and $T(r, t, R(t))$, in which $R(t)$ is a term like $f(t)+\tau g(t)$, and where $f(t)$ and $g(t)$ are smoothed functions which can substitute $H(t)$ and $\delta(t)$, respectively.

The analytical solution of (12) is obtained by the Laplace transform. From previous results $([17],[18])$ this solution can be expressed as follows:

$$
T(r, t, D(t))=T_{0}+\frac{P}{4 \pi k r_{0}} V\left(\frac{r}{r_{0}}, \frac{\alpha t}{r_{0}^{2}}, d\left(\frac{\alpha t}{r_{0}^{2}}\right)\right),
$$

with

$$
V(\rho, \xi, d(\xi))=F(\xi) * d(\xi))
$$

where $*$ is the convolution operator, $d(\xi)$ is the dimensionless term of $D(t)$, i.e. $D\left(\frac{r_{0}^{2} \xi}{\alpha}\right)$ and

$$
\begin{aligned}
F(\xi) & =\mathfrak{L}_{s}^{-1}\left[\frac { 1 } { 2 \rho } \left(\int_{\rho}^{\infty} \frac{e^{-\sqrt{\lambda s^{2}+\zeta s+b}(u-\rho)}}{\sqrt{\lambda s^{2}+\zeta s+b}} \frac{d u}{u^{3}}\right.\right. \\
& +\int_{1}^{\rho} \frac{e^{-\sqrt{\lambda s^{2}+\zeta s+b}(\rho-u)}}{\sqrt{\lambda s^{2}+\zeta s+b}} \frac{d u}{u^{3}}-\int_{1}^{\infty} \frac{e^{-\sqrt{\lambda s^{2}+\zeta s+b}(u+\rho-2)}}{\sqrt{\lambda s^{2}+\zeta s+b}} \frac{d u}{u^{3}} \\
& \left.\left.+\int_{1}^{\infty} \frac{6 \sqrt{\lambda s^{2}+\zeta s+b}}{m\left(\lambda s^{2}+s\right)+3 \sqrt{\lambda s^{2}+\zeta s+b}+3} \frac{e^{-\sqrt{\lambda s^{2}+\zeta s+b}(u+\rho-2)}}{\sqrt{\lambda s^{2}+\zeta s+b}} \frac{d u}{u^{3}}\right)\right]
\end{aligned}
$$

$\lambda=\frac{\alpha \tau}{r_{0}^{2}}$ being the dimensionless relaxation time, $\rho=\frac{r}{r_{0}}$ the dimensionless variable $r$, $b=\frac{B r_{0}^{2}}{\alpha}$ the dimensionless constant of the blood perfusion term and $m=\frac{\eta_{0} c_{0}}{\eta c}$ the dimensionless electrode thermal inertia.

In order to reach a general conclusion about which smoothed functions could be candidates to substitute the Heaviside function and Dirac's delta distribution, we first try to compare two generic solutions $T(r, t, D 1(t))$ and $T(r, t, D 2(t))$, where $D 1(t)$ and $D 2(t)$ are different distributions.

From the previous sections,

$$
V(\rho, \xi, d 1(\xi))-V(\rho, \xi, d 2(\xi))=F(\xi) *(d 1(\xi)-d 2(\xi))
$$

where $d 1(\xi)=D 1\left(\frac{r_{0}^{2} \xi}{\alpha}\right)$ and $d 2(\xi)=D 2\left(\frac{r_{0}^{2} \xi}{\alpha}\right)$. To quantify this difference we consider a property of the convolution of two distributions of $\mathcal{D}^{\prime}(\mathbb{R})$, if at least one of them has compact support (see Annex). 
We denote the space of the indefinitely differentiable functions in $\mathbb{R}$ (endowed with its "usual Fréchet topology", i. e. metrizable and complete) by $\mathcal{C}^{\infty}(\mathbb{R})$. From Schwartz's Theory of Distributions [24] if $D \in \mathcal{D}^{\prime}(\mathbb{R})$ has compact support and $h \in \mathcal{C}^{\infty}(\mathbb{R})$, the convolution operator $D * h$ belongs to $\mathcal{C}^{\infty}(\mathbb{R})$. Moreover, if we set $h \in \mathcal{C}^{\infty}(\mathbb{R})$, the map: $\mathcal{D}^{\prime}(\mathbb{R}) \rightarrow \mathcal{C}^{\infty}(\mathbb{R})$ such that $D \rightarrow D * h$ is continuous in the sense that if $\left\{D_{n}, n \in \mathbb{N}\right\}$ is a sequence of distributions with compact support which converges to $D$ in $\mathcal{D}^{\prime}(\mathbb{R})$, then $\left\{D_{n} * h, n \in \mathbb{N}\right\}$ converges to $D * h$ in $\mathcal{C}^{\infty}(\mathbb{R})$.

In other words, if the distributions $D 1(t)$ and $D 2(t)$ are "close enough" in $\mathcal{D}^{\prime}(\mathbb{R})$ and $D 1(t)-D 2(t)$ has compact support, then the difference $V(\rho, \xi, d 1(\xi))-V(\rho, \xi, d 2(\xi))$ can be "sufficiently small", and hence $T(r, t, D 1(t))-T(r, t, D 2(t))$ can also be "sufficiently small". This would be the condition necessary in order to consider $D_{1}(t)$ a good substitute for $D_{2}(t)$ and viceversa.

In the case of the HBE, we can apply the above result taking:

a) $h=F(\xi) \in \mathcal{C}^{\infty}(\mathbb{R})$.

b) $G_{n}(\xi)=H(\xi)-f_{n}(\xi)+\lambda\left(\delta(\xi)-g_{n}(\xi)\right)$, choosing $f_{n}(\xi)$ and $g_{n}(\xi)$ such that $(H(\xi)-$ $f_{n}(\xi)+\lambda\left(\delta(\xi)-g_{n}(\xi)\right)$ is a distribution of $\mathcal{D}^{\prime}(\mathbb{R})$ with compact support, the sequence $\left\{f_{n}(\xi) ; n \in \mathbb{N}\right\}$ converges to $H(\xi)$ in $\mathcal{D}^{\prime}(\mathbb{R})$ and the sequence $\left\{g_{n}(\xi) ; n \in \mathbb{N}\right\}$ converges to $\delta(\xi)$ in $\mathcal{D}^{\prime}(\mathbb{R})$. Then $\left\{G_{n}, n \in \mathbb{N}\right\}$ is a sequence of distributions with compact support which converges to zero in $\mathcal{D}^{\prime}(\mathbb{R})$.

As the map $\mathcal{D}^{\prime}(\mathbb{R}) \rightarrow \mathcal{C}^{\infty}(\mathbb{R})$ such that $G \rightarrow G * h$ is continuous, then $\left\{G_{n} * h, n \in \mathbb{N}\right\}$ converges to zero in $\mathcal{C}^{\infty}(\mathbb{R})$. That is, $\left\{V(\rho, \xi, H(\xi)+\lambda \delta(\xi))-V\left(\rho, \xi, f_{n}(\xi)+\lambda g_{n}(\xi)\right), n \in \mathbb{N}\right\}$ converges to zero in $\mathcal{C}^{\infty}(\mathbb{R})$ for every $\rho>1$. Hence $\left\{V\left(\rho, \xi, f_{n}(\xi)+\lambda g_{n}(\xi)\right), n \in \mathbb{N}\right\}$ converges to $V(\rho, \xi, H(\xi)+\lambda(\xi))$ in $\mathcal{C}^{\infty}(\mathbb{R})$ for every $\rho>1$. Then for $n \in \mathbb{N}$ "large enough", the analytical solution $T\left(r, t, f_{n}(t)+\tau g_{n}(t)\right)$ is "close" to the analytical solution of the original HBE, $T(r, t, H(t)+\tau \delta(t))$.

\section{II.2 Smoothed functions}

There are several smoothed functions which could be candidates to substitute Heaviside $H(t)$ and Dirac's delta distribution $\delta(t)$ in the HBE. One of the simplest options could be to use a logistic function $f(t)=\frac{1}{1+e^{-p t}}$ as substitute for $H(t)$ and its derivative $g(t)=f^{\prime}(t)$ as substitute for $\delta(t)$ (see Fig. 1A). Another option is to use a probabilistic Gaussian function $h(t)=\frac{1}{q \sqrt{2 \pi}} e^{\frac{-t^{2}}{2 q^{2}}}$, instead of the derivative function $f^{\prime}(t)$, as a substitute for $\delta(t)$ (see Fig. 1B).

Still other options could use polynomial interpolation functions built from pairs of 
values by means of the splines method. This would provide many candidate functions $f(t)=I H(t)$ and $g(t)=I D(t)$ to substitute $H(t)$ and $\delta(t)$ respectively. An example of this kind of function are the families of smoothed functions with a continuous second derivative without overshoot, denoted by $f l c 2 h s(t, p)$ and $f l d c 2 h s(t, q)$ (where $p$ and $q$ are parameters), which are used by COMSOL Multiphysics (COMSOL, Burlington, MA, USA), as approximations of $H(t)$ and $\delta(t)$ respectively (see Fig. 2). This software is being increasingly used to study the heating of biological tissue with the BE ([20],[22], [23]), .

\section{II.3 Description of the numerical model}

In order to put the above ideas into practice we considered a theoretical RFA model using the HBE. The liver was chosen as the target tissue, with the following characteristics: Density $\eta=1060 \mathrm{~kg} / \mathrm{m}^{3}$, specific heat $c=3600 \mathrm{~J} / \mathrm{kg} \cdot \mathrm{K}$ and thermal conductivity $k=$ $0.502 \mathrm{~W} / \mathrm{m} \cdot \mathrm{K}$. The blood properties were density $\eta_{b}=1000 \mathrm{~kg} / \mathrm{m}^{3}$ and specific heat $c_{b}=4148 \mathrm{~J} / \mathrm{kg} \cdot \mathrm{K}$. Electrode characteristics were radius $r_{0}=1.5 \mathrm{~mm}$, density $\eta_{0}=21500$ $\mathrm{kg} / \mathrm{m}^{3}$ and specific heat $c_{0}=132 \mathrm{~J} / \mathrm{kg} \cdot \mathrm{K}$. The perfusion coefficient was $w_{b}=0.01 \mathrm{~s}^{-1}$, which is slightly higher than the maximum value proposed by Chang and Nguyen [21], corresponding to a well-perfused organ. Blood temperature and initial tissue temperature were $T_{0}=37^{\circ} \mathrm{C}$. There is a lack of experimental data regarding the thermal relaxation time $\tau$ of biological tissue. In fact, although Mitra et al [26], found the value to be $\tau=16 \mathrm{~s}$ in processed meat, no values have been measured for non excised tissues. Here we assumed that $\tau=16 \mathrm{~s}$. The applied power was $P=1 \mathrm{~W}$.

In order to study the suitability of the different functions $f(t)$ and $g(t)$ mentioned in Section II.2, we obtained the numerical solutions of the HBE using each one, i.e. $T(r, t, f(t)+\tau g(t))$ by means of COMSOL. This numerical approximation is denoted by $N T(r, t, f(t)+\tau g(t))$. We then studied the temperature evolution at $r=2 r_{0}$ for $160 \mathrm{~s}$ of RFA, and used Mathematica 7.0 (Wolfram Research, Champaign, IL, USA) to plot the analytical solutions.

\section{Results}

\section{III.1 Logistic function and its derivative}

We first conducted an analysis in order to choose the most suitable $p$ parameter of the logistic function. We plotted the logistic function for different $p$ values ranging, from 0.05 to 5 (see Fig. 1A). The approximation of the logistic function towards $H(t)$ improves 
when $p$ increases. Thus, we chose $p=50$, which was the greatest $p$ value we used in Fig. 1A. However, we observed that for $p \geq 5$ the approximation of the logistic function to the Heaviside function was suitable. Fig. 3 shows the temperature evolution for $p$ between 0.05 and 50. In this case $p=5$ and $p=50$ provided a temperature plot very similar to that obtained with the analytical solution using $H(t)$, as will be shown in Section III.4.

\section{III.2 Logistic function and probabilistic Gaussian function}

In this case we searched for the optimum value of $q$ in the probabilistic Gaussian function. We plotted the Gaussian function for different $q$ values ranging, from 0.05 to 50 (See Fig. 1B). In this case, the approximation of the gaussian function towards $\delta(t)$ improves when $q$ decreases, however, for very small values the numerical method fails. We then selected $q=0.05$ which was the minimum $q$ value we tested and the approximation was suitable. Fig. 4 shows temperature evolution for different values of $q, p=50$ being. In this case $q=0.05$ (solid line) provided a temperature plot very similar to that obtained with the analytical solution using $H(t)$, as will be shown in Section III.4.

\section{III.3 Smoothed functions of COMSOL Multiphysics}

The smoothed functions used by COMSOL to substitute $H(t)$ and $\delta(t)$ also have a couple of parameters $p$ and $q$. We conducted a similar analysis to choose their optimum values. We found that large variations in $p$ did not give a noticeable differences in the results, while they were highly influenced by variations in $q$. The optimum value of $q=$ 0.035 was found; the numerical method always failed with values other than this one. Fig. 5 shows temperature evolution for different values of $p, q=0.035$ being. Note that the results did not vary much when $p$ changed, even in a broad range.

\section{III.4 Comparison between candidate functions}

Fig. 6 compares the temperature evolution of all the candidate functions considered and their optimum values for parameters $p$ and $q$ as obtained in the previous steps. Fig. 6 also includes (solid line) the analytical solution using $H(t)$ and $\delta(t)$ directly, which can be considered as the standard to check the suitability of each pair of candidate functions.

\section{Discussion}

This study was conducted to identify the types of smoothed functions that could be possible candidates to substitute $H(t)$ and $\delta(t)$ in problems involving the HBE. Several 
functions were considered and compared with the numerical HBE solutions. Only COMSOL smoothed functions provided a solution almost identical to the analytical one. Here we provide a mathematical explanation for this.

The theoretical basis presented in the Annex proves that if $f(t)$ and $g(t)$ are "close to" $H(t)$ and $\delta(t)$ respectively and $H(t)-f(t)$ and $\delta(t)-g(t)$ have compact support, then we can ensure that $T(r, t, H(t)+\tau \delta(t))$ is "close to" $T(r, t, f(t)+\tau g(t))$, i.e. $f(t)$ and $g(t)$ are good candidates to substitute $H(t)$ and $\delta(t)$ respectively. Otherwise if $H(t)-f(t)$ or $\delta(t)-g(t)$ does not have compact support, we are not able to reach a conclusion on the suitability of the candidate functions.

It is necessary to emphasize the essential difference between the logistic function and an interpolation function $I H(t)$ as used in COMSOL. A properly calculated interpolation function can be zero for $t<-a$ and is 1 for $t>a$ for some $a>0$. In contrast, in the logistic functions the lines $y=0$ and $y=1$ are asymptotes (see Fig. 1A). Likewise, the main difference between the probabilistic Gaussian function or the derivative of the logistic function, and $I D(t)$ is that $I D(t)$, properly calculated, can be zero for $|t|>b$, for some $b>0$, while in the others the line $y=0$ is an asymptote (see $1 \mathrm{~B}$ and $\mathrm{C}$ ). In general, since $H(t)-I H(t)$ and $\delta(t)-I D(t)$ have compact support, $N T(r, t, I H(t)+\tau I D(t))$ is not only a numerical approximation of $T(r, t, I H(t)+\tau I D(t))$ but also of $T(r, t, H(t)+\tau \delta(t))$.

COMSOL functions $f l c 2 h s(t, p)$ and $f l d c 2 h s(t, q) \in \mathcal{D}^{\prime}(\mathbb{R}), \forall p, q \in \mathbb{R}^{+}$and satisfy that $\lim _{p \rightarrow 0}(H(t)-f l c 2 h s(t, p))=0$ and $\lim _{q \rightarrow 0}(\delta(t)-f l d c 2 h s(t, 0))=0$ in $\mathcal{D}^{\prime}(\mathbb{R})$. Moreover the parameter $p$ controls the value of $a$ such that $f l c 2 h s(t, p)$ is zero for $t<-a$ and it is 1 for $t>a$. And likewise the parameter $q$ controls the number $b$ such that $f l d c 2 h s(t, q)$ is zero for $|t|>b$. Consequently, we can affirm that the numerical solution $N T(r, t, f l c 2 h s(t, p)+$ $\tau f l d c 2 h s(t, q))$ is not only an approximation of $T(r, t, f l c 2 h s(t, p)+\tau f l d c 2 h s(t, q))$, but also of $T(r, t, H(t)+\tau \delta(t))$.

Otherwise, if $f(t)$ is a logistic function and $g(t)$ is the probabilistic Gaussian function or the derivative of the logistic function, neither $H(t)-f(t)$ nor $\delta(t)-g(t)$ have compact support. Accordingly, $N T(r, t, f(t)+\tau g(t))$ is a numerical approximation of $T(r, t, f(t)+$ $\tau g(t))$, but we cannot say whether it is a numerical approximation of $T(r, t, H(t)+\tau \delta(t))$ or is not.

It is also important to note that in the expression of the analytical solution of the HBE

$$
V(\rho, \xi, H(\xi)+\lambda \delta(\xi))=F(\xi) *(H(\xi)+\lambda \delta(\xi))
$$

neither $F(\xi)$ nor $H(\xi)+\lambda \delta(\xi)$ have compact support. This explains why Figure 6 presents 
a peak. In this case $T(r, t, H(t)+\lambda \delta(t))$ inherits the irregularity of $H(t)+\lambda \delta(t)$, and $T(r, t, H(t)+\lambda \delta(t)) \notin \mathcal{C}^{\infty}(\mathbb{R})$.

The very simple geometry included in the modeling could be considered as a limitation of the study. In fact, in problems with the same governing equation but with other geometries, the convolution factor $F(\xi)$ will be different. In spite of this, $H(\xi)+\lambda \delta(\xi)$ is implicit to the external energy source. Consequently, by choosing appropiate $f_{n}(t)$ and $g_{n}(t), n \in \mathbb{N}$, the corresponding difference $T(r, t, H(t)+\tau \delta(t))-T\left(r, t, f_{n}(t)+\tau g_{n}(t)\right)$ tends to zero in $\mathcal{C}^{\infty}(\mathbb{R})$ for every $r>r_{0}$, so that the conclusion that the interpolation functions are as suitable substitutes for $H(t)$ and $\delta(t)$ in HBE is still valid.

In addition, the findings of this study open the way for other more general situations. For instance, if a model of partial differential equations has a distribution $D$, in many cases it can be suitably substituted by an approximate smoothed function $h \in \mathcal{D}^{\prime}(\mathbb{R})$ of $D$ such that $D-h$ has compact support.

\section{Conclusions}

The numerical solutions of HBE showed that only the families of smoothed functions with a continuous second derivative without overshoot used by COMSOL Multiphysics provided solutions similar to those obtained analytically from the original Heaviside and Delta distributions. With the other candidate functions, such as the logistic function as substitute for the Heaviside function and its derivative, and the probabilistic Gaussian function as substitute for the Delta distribution, we obtained approximate solutions to the analytical, but not as satisfactory as the obtained with COMSOL. From the mathematical analysis we concluded that in order to find a suitable smoothed function $(f)$ to substitute any mathematical distribution $(D)$ in the HBE, the difference $D-f$ has to have compact support. In fact, of all the smoothed functions considered here, only the smoothed COMSOL Multiphysics functions accomplish this requeriment. 


\section{Annex}

Dirac's Delta was introduced to satisfy the need of Quantum Mechanics for a measure of mass placed at a point $x_{0}$ denoted here by $\delta_{x_{0}}$. It is defined in the space of continuous functions with compact support $\mathcal{C}_{0}^{0}(\mathbb{R})$ such that,

i) $<\delta_{x_{0}}, f>=f\left(x_{0}\right), \quad \forall f \in \mathcal{C}_{0}^{0}(\mathbb{R})$.

ii) $\delta_{x_{0}}(\mathbb{R})=1$.

A measure $\mu$ has a density function with respect to the Lebesgue measure if there is a function $\phi_{\mu}$ integrable in the compact subsets of $\mathbb{R}$, such that:

i) $<\mu, f>=\int_{\mathbb{R}} \phi_{\mu}(x) f(x) d x$.

ii) $\mu(A)=\int_{A} \phi_{\mu}(x) d x$, for all measurable set $A \subset \mathbb{R}$.

In this case we identify the measure $\mu$ with its density function $\phi_{\mu}$, and then we say that the measure $\mu$ is also a function.

For example, for every $\varepsilon>0$, given the interval $I_{\varepsilon}=[0, \varepsilon]$ we can define the measure $\mu_{\varepsilon}$ such that $\mu_{\varepsilon}(A)=\frac{1}{\varepsilon} \int_{A \cap I_{\varepsilon}} d x$. We consider the function $\phi_{\varepsilon}(x)=\frac{1}{\varepsilon}$ if $x \in I_{\varepsilon}$ and zero if $x \notin I_{\varepsilon}$. It is easy to see that $\phi_{\varepsilon}$ is the density of $\mu_{\varepsilon}$. Moreover $\mu_{\varepsilon}(\mathbb{R})=1$. In fact, we put the constant factor $\frac{1}{\varepsilon}$ in the definition only because of this probabilistic requirement.

Sometimes Dirac's Delta function is defined in $x_{0}$ as the function $x \rightarrow \delta\left(x-x_{0}\right)$, such that it is infinity at $x=x_{0}$ and zero if $x \neq x_{0}$. Then, on identifying the measure $\delta_{x_{0}}$ with $\delta\left(x-x_{0}\right)$; that is, as if $\delta\left(x-x_{0}\right)$ was the density of the measure $\delta_{x_{0}}$.

With this identification the following conditions should be accomplished:

i) $\quad<\delta_{x_{0}}, f>=f\left(x_{0}\right)=\int_{\mathbb{R}} f(x) \delta\left(x-x_{0}\right) d x$, for every continuous function $f$ of compact support.

ii) $\quad 1=\int_{\mathbb{R}} \delta\left(x-x_{0}\right) d x$.

However, from the classical theory of real functions, the integral of a function which is zero in all real numbers except in $x_{0}$ must be zero, which contradicts $i$ ) and $i i$ ). In consequence, $\delta\left(x-x_{0}\right)$ is not a function. Then we conclude that Dirac's Delta is a positive measure without a density function, hence it is not a function. Furthermore, it is the simplest example of measure which is not a function (without density).

Schwartz's Distribution Theory provides theoretical support. We consider the space $\mathcal{D}(\mathbb{R})$ of indefinitely differentiable functions with compact support, endowed with a "certain" topology. The distribution space in the sense of Schwartz is the topological dual of $\mathcal{D}(\mathbb{R})$ (i.e., the space of linear and continuous forms defined in $\mathcal{D}(\mathbb{R})$ ) endowed with the weak topology $\left(\right.$ denoted by $\left.\mathcal{D}^{\prime}(\mathbb{R})\right)$. 
Three facts have to be taken into account:

1) $\mathcal{D}^{\prime}(\mathbb{R})$ contains functions (for example, measures with densities) and mathematical objects which are not functions, for example, Dirac's Delta. But a Schwartz's distribution is always a linear and continuous form defined in $\mathcal{D}(\mathbb{R})$.

2) Every integrable function $f$ in the compact sets of $\mathbb{R}$ defines a distribution, and thus can also be considered also as a distribution. For example, the Heaviside function $H_{x_{0}}$ is also a distribution.

3) In the space $\mathcal{D}^{\prime}(\mathbb{R})$ it is possible to extend the notion of derivative, which coincides with the derivation of functions if the distribution is a derivable function. And the derivative of a distribution $T$, denoted $D T$, is also a distribution. Bydefinition, if $T \in \mathcal{D}^{\prime}(\mathbb{R})$, $D T$ is the element of $\mathcal{D}^{\prime}(\mathbb{R})$ such that for every $f \in \mathcal{D}(\mathbb{R}),\langle D T, f\rangle:=-\langle T, D f\rangle$. In particular, the Heaviside function $H_{x_{0}}$, as a function, is not derivable in $x_{0}$, but as distribution $D H_{x_{0}}=\delta_{x_{0}}$. Obviously this property has been used to obtain the source term for the governing equation. 


\section{References}

[1] Berjano E J: Theoretical modeling for radiofrequency ablation: state-of-the-art and challenges for the future. Biomed Eng Online, 2006, 5, 24.

[2] Pennes H H: 1998 Analysis of tissue and arterial blood temperatures in the resting human forearm. J Appl Physiol, 1948, 85(1): 5-34.

[3] Liu J, Chen X and Xu L X: New thermal wave aspects on burn evaluation of skin subjected to instantaneous heating. IEEE Trans Biomed Eng, 1999, 46: 420-8.

[4] Özisik M N and Tzou D T: On the wave theory in heat conduction. J Heat Transfer, 2004, 116: 526-35.

[5] Shih T C, Kou H S, Liauh C T and Lin W L: The impact of thermal wave characteristics on thermal dose distribution during thermal therapy: a numerical study. Med Phys, 2005, 32(9): 3029-36.

[6] F.S. Loureiro, P. Oyarzún, J.P.L. Santos, W.J. Mansur, and C.A.B. Vasconcellos. A hybrid time/Laplace domain method based on numerical green's functions applied to parabolic and hyperbolic bioheat transfer problems. Mecánica Computacional Vol. XXIX, pp 5599-5611.

[7] F. Xu, T.J. Lu. Skin biothermomechanics: Modeling and experimental characterization. In Advances in Applied Mechanics, vol. 43. Edited by H Aref and E Van der Hiessen. Elsevier, 2009.

[8] Ahmadikia, H.; Fazlali, R.; Moradi, A. Analytical solution of the parabolic and hyperbolic heat transfer equations with constant and transient heat flux conditions on skin tissue. International Communications in Heat and Mass Transfer vol. 39 issue 1 January, 2012. p. 121-130.

[9] F. Xu, M.Lin, T.J.Lu. Modeling skin thermal pain sensation: Role of non-Fourier thermal behavior in transduction process of nociceptor. Computers in Biology and Medicine 40 (2010) 478-486.

[10] Jing Liu, Xuexue Zhang, Cuncheng Wang, Wenqiang Lu and Zepei Ren. Generalized time delay bioheat equation and preliminary analysis on its wave nature. Chinese Science Bulletin. Volume 42, Number 4 (1997), 289-292. 
[11] Gou Chenhua and Cai Ruixian. An analytical solution of non-Fourier Chen-Holmes bioheat transfer equation. Chinese Science Bulletin. Volume 50, Number 23 (2005), 2791-2792.

[12] P. R. Sharma, Sazid Ali, V. K. Katiyar. Numerical study of heat propagation in living tissue subjected to instantaneous heating. Indian Journal of Biomechanics: Special Issue (NCBM 7-8, March 2009), 205-209.

[13] Kuo-Chi Liu, Po-Jen Cheng, Yan-Nan Wang. Analysis of non-fourier thermal behaviour for multi-layer skin model. Thermal Science, 2011, Vol. 15, Suppl. 1, pp. S61-S67.

[14] 12. Xu, F., Seffen, K. A., Lu, T. J., Non-Fourier analysis of skin biothermomechanics. Int. J. Heat and Mass Transfer, 51 (2008), 9-10, pp. 2237-2259.

[15] 13. Liu, K. C., Cheng, P. J. Finite propagation of heat transfer in a multi-layer tissue. AIAA Journal of Thermophysics and Heat Transfer. 22 (2008), 4, pp. 775-782.

[16] Catteneo C: Sur une forme de l'équation de la chaleur éliminant le paradoxe d'une propagation instantaneé. Compes Rendus, 1958, 247: 431-3.

[17] López Molina J A, Rivera M J, Trujillo M and Berjano E J: Effect of the thermal wave in radiofrequency ablation modeling: An analytical study. Phys Med Biol, 2008, $53(5): 1447-62$.

[18] López Molina J A, Rivera M J, Trujillo M and Berjano E J: Thermal modeling for pulsed radiofrequency ablation: Analytical study based on hyperbolic heat equation. Med Phys, 2009, 36(4): 1112-9.

[19] Erez A and Shitzer A: Controlled destruction and temperature distributions in biological tissues subjected to monoactive electrocoagulation. J Biomech Eng, 1980, 102: 42-9.

[20] Barauskas R, Gulbinas A, Vanagas T and Barauskas G: Finite element modeling of cooled-tip probe radiofrequency ablation processes in liver tissue. Comput Biol Med, 2008, 38(6): 694-708.

[21] Chang I and Nguyen U D: Thermal modeling of lesion growth with radiofrequency ablation devices. Biomed Eng Online, 2004, 3(1): 27. 
[22] Ekstrand V, Wiksell H, Schultz I, Sandstedt B, Rotstein S and Eriksson A: Influence of electrical and thermal properties on RF ablation of breast cancer: is the tumour preferentially heated?. Biomed Eng Online, 2005, 11: 4-41.

[23] Quaranta V, Manenti G, Bolacchi F, Cossu E, Pistolese C A, Buonomo O C, Carotenuto L, Piconi C and Simonetti G: FEM analysis of RF breast ablation: multiprobe versus cool-tip electrode. Anticancer Res, 2007, 27(2): 775-84.

[24] Dautray R and Lions JJ: Analyse Mathématique et calcul numérique. Masson Editores, 1988, 4, Anexe 1 and 2.

[25] Bilotsky Y and Gasik M: Modelling Multilayers Systems with Time-Depended Heaviside and New Transition Functions. Proceedings of the 2006 Nordic COMSOL Conference.

[26] Mitra K, Kumar S, Vedavarz A and Moallemi M K: Experimental evidence of hyperbolic heat conduction in processes meat. J Heat Transfer, 1995, 117: 568-73. 

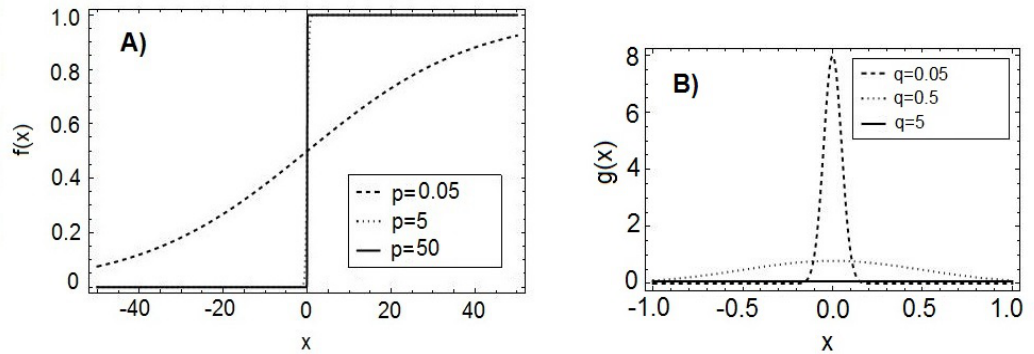

Figure 1: (A) Three logistic functions $f(t)=\frac{1}{1+e^{-p t}}$ as possible smoothed functions to substitute the Heaviside function $H(t)$ and (B) Three gaussian functions $g(t)=\frac{1}{q \sqrt{2 \pi}} e^{-\frac{t^{2}}{2 q^{2}}}$ as possible smoothed functions to substitute the Dirac distribution $\delta(t)$. 


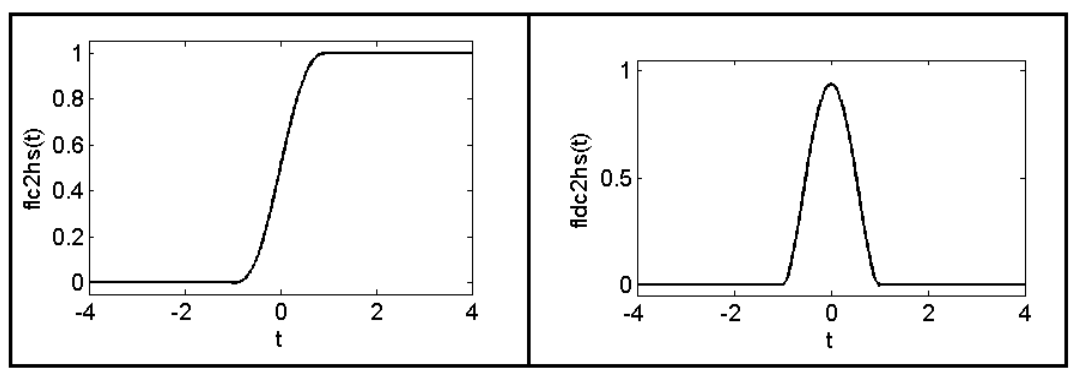

Figure 2: Representation of $f l c 2 h s(t, p)$ with $p=1$ (left) and $f l d c 2 h s(t, q)$ with $q=1$ (right). These smoothed functions are used by COMSOL Multiphysics as candidates to substitute Heaviside and Dirac distributions, respectively. 


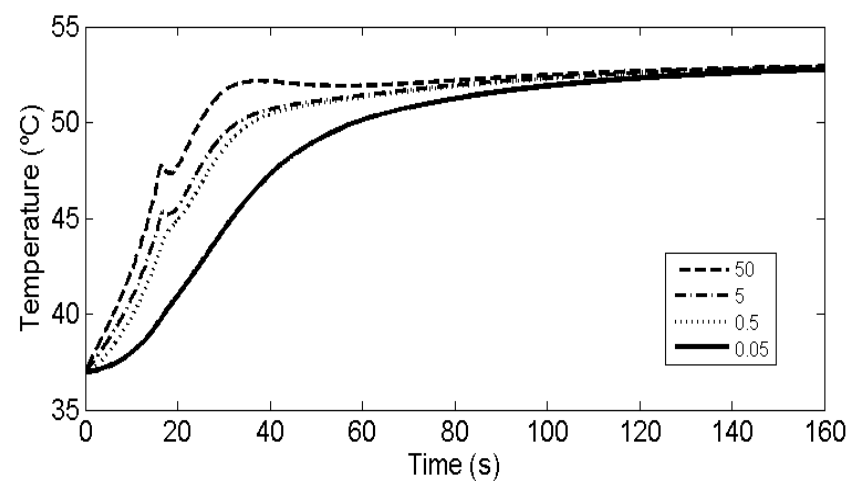

Figure 3: Temperature evolution at $r=2 r_{0}$ for different values of $p$ of the logistic function. This function and its derivative were used as candidates to substitute Heaviside and Dirac distributions, respectively. 


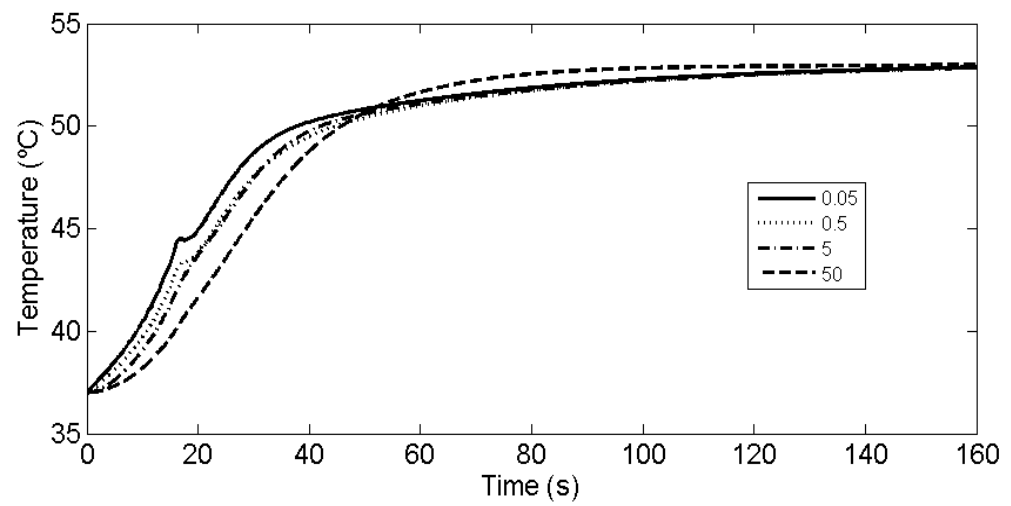

Figure 4: Temperature evolution at $r=2 r_{0}$ for different values of $q$ of the probabilistic Gaussian function, $p=50$ being in the logistic function. These functions were used as candidates to substitute Dirac and Heaviside distributions, respectively. 


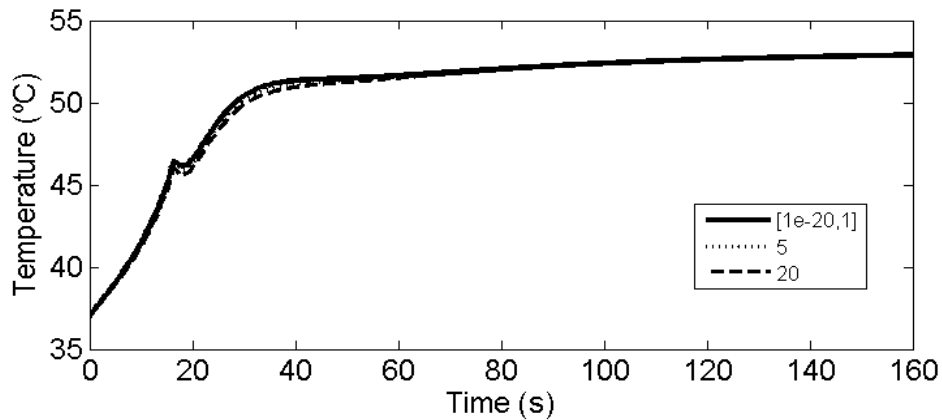

Figure 5: Temperature evolution at $r=2 r_{0}$ for different values of $p$ of the smoothed functions used by COMSOL Multiphysics, $q=0.035$ being. These functions were used as candidates to substitute Dirac and Heaviside distributions. 


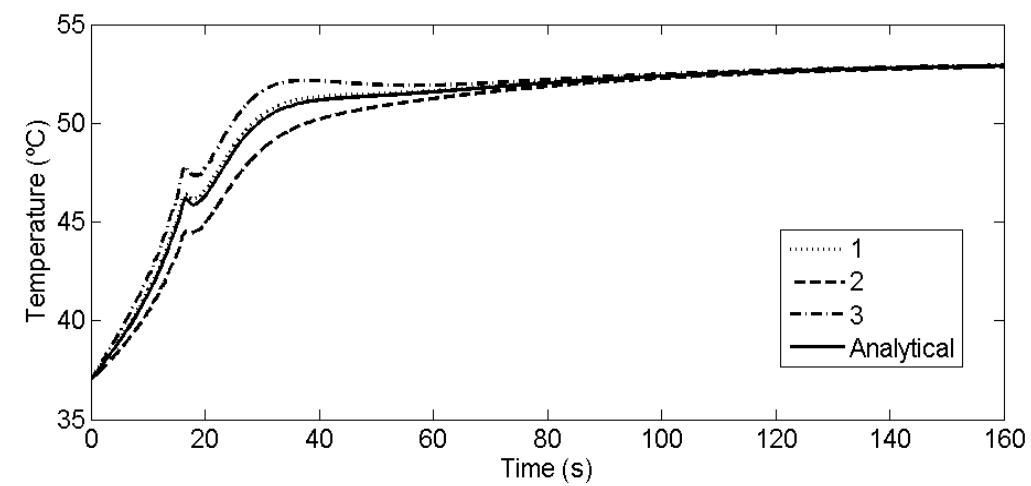

Figure 6: Temperature evolution at $r=2 r_{0}$ for the different candidate functions to substitute Dirac and Heaviside distributions. Solid line corresponds with the analytical solution using directly $H(t)$ and $\delta(t)$, which can represent the comparison standard. 1) COMSOL smoothed functions, 2) Logistics and Gaussian probabilistic functions, and 3) Logistic functions and its derivative. 